\section{Subcutaneous Tocilizumab May Be Less Effective than Intravenous Tocilizumab in the Treatment of Juvenile Idiopathic Arthritis-associated Uveitis}

\section{To the Editor:}

Tocilizumab (TCZ) is a fully humanized, monoclonal, anti-interleukin-6R antibody shown by clinical trials to be a safe and effective treatment for patients with rheumatoid arthritis (RA) or systemic-onset or polyarticular juvenile idiopathic arthritis (JIA) $)^{1,2,3}$. TCZ seems to be an effective agent to treat JIA-associated uveitis, and several case reports have described a good efficacy profile in patients with refractory JIA-associated uveitis ${ }^{4,5}$. Interim outcomes of the STOP-Uveitis clinical trial were presented by Nguyen, et al, showing that TCZ has a good safety and efficacy profile in noninfectious uveitis ${ }^{6}$. For several years, TCZ has also been available in a subcutaneous (SC) formulation, and switching from intravenous (IV) to SC administration may provide greater flexibility and quality of life to patients who follow TCZ treatment. Several clinical trials have shown the safety and efficacy profile of TCZ-SC to be comparable to that of TCZ-IV ${ }^{7}$. Switching from TCZ-IV to SC was shown to be generally successful and sustained over time ${ }^{8}$. A recent study analyzed clinical data from 58 patients with RA who switched from TCZ-IV to TCZ-SC, suggesting that the switch in realworld settings is effective and can be considered for convenience ${ }^{9}$. In that study, only the disease activity of high-body-weight patients showed a tendency to worsen after switching. At present, TCZ-SC is being tested in patients with JIA in 2 ongoing phase Ib clinical trials (NCT01904292 and NCT01904279, www.clinicaltrials.gov) and in 1 phase II clinical trial, currently recruiting in the UK, investigating the efficacy of TCZ-SC in JIA-associated uveitis that is refractory to anti-tumor necrosis factor (www.isrctn.com/ISRCTN95363507). To our knowledge, there are no reports on the results of switching from TCZ-IV to TCZ-SC in patients with JIA.

We describe 4 patients with longterm JIA and associated chronic anterior uveitis (CAU) who had good and sustained clinical response with TCZ-IV and who experienced early flares after switching to TCZ-SC. Our study received ethics approval from the Clinical Research Ethics Committee of Vall d'Hebron University Hospital and Research Institute.
Table 1 describes the clinical characteristics of these patients at baseline. Three of the patients (patients 1,3,4) experienced bilateral CAU and patient 2, unilateral CAU. In these patients, TCZ was switched from IV to SC mainly for patient convenience. All patients were in clinical remission on TCZ-IV treatment, had a stable clinical situation, and had been receiving TCZ-IV for an average of 3.4 years (range, $2-5 \mathrm{yrs}$ ) before switching. Three of the patients (patients $1,2,3$ ) received a TCZ-IV dosage of $8 \mathrm{mg} / \mathrm{kg} / 4$ weeks, whereas patient 4 received $8 \mathrm{mg} / \mathrm{kg} / 2$ weeks of TCZ-IV. Three of these patients (patients 1,2,3) did not experience disease flare during TCZIV treatment, and only 1 (patient 4 ) had a mild uveitis flare that was promptly controlled with corticosteroid eyedrops. Patients 1 and 3 had experienced a severe CAU course in the past, presenting chronic severe ocular complications and severe visual impairment as a consequence of the disease course. In one of these patients (patient 3), the eye examination was hindered by synechiae, media opacity, and calcifications, but the ophthalmologist reported improvement during TCZ-IV treatment. In addition, 2 of them (patients 1,2 ) required several surgical procedures for joint complications before starting TCZ treatment.

Three of our 4 patients (patients 1,3,4) started TCZ-SC at doses of 162 $\mathrm{mg} /$ week. One of our patients (patient 2) weighed $32 \mathrm{~kg}$ and started TCZ$\mathrm{SC}$ adjusted to body surface area at $108 \mathrm{mg} /$ week, as usual in clinical practice. All patients experienced disease relapse a few months after TCZSC was started (Table 2) ${ }^{10,11}$ and had no changes in concomitant medications while receiving TCZ-IV or TCZ-SC. In all cases, TCZ-SC was discontinued owing to a lack of efficacy: 3 of them (patients $2,3,4$ ) for ocular flare and 2 (patients 1,3) for joint flare. One of the patients (patient 3) who experienced joint flare also had the most severe ocular condition, and simultaneously with the joint symptoms, experienced vitreous hemorrhage, an eye complication that could be interpreted as related to ocular inflammation. The patients did not experience any other adverse event while receiving TCZ-SC treatment.

All 4 patients with JIA who switched to TCZ-SC while in remission with TCZ-IV treatment experienced a flare (ocular and/or joint) in the first few months after TCZ-SC initiation. Our observation suggests that the results previously observed in patients with RA cannot be extrapolated to patients with JIA. The pathogenesis of ocular involvement in JIA is not yet fully

Table 1. Demographics and main clinical characteristics of patients with juvenile idiopathic arthritis (JIA).

\begin{tabular}{|c|c|c|c|c|c|c|c|c|}
\hline Patient & Age/Sex & $\begin{array}{c}\text { ILAR } \\
\text { Classification }\end{array}$ & $\begin{array}{l}\text { ANA/RF/ } \\
\text { HLA-B27 }\end{array}$ & $\begin{array}{l}\text { Age at JIA } \\
\text { Diagnosis, } \\
\text { yrs }\end{array}$ & $\begin{array}{c}\text { Age at } \\
\text { Uveitis } \\
\text { Onset, yrs }\end{array}$ & $\begin{array}{l}\text { Duration of } \\
\text { Uveitis, yrs }\end{array}$ & $\begin{array}{c}\text { Secondary Ocular Complications } \\
\text { and Procedures }\end{array}$ & $\begin{array}{l}\text { Previous } \\
\text { DMARD }\end{array}$ \\
\hline 3 & $18 / \mathrm{F}$ & $\begin{array}{l}\text { Oligoarticular } \\
\text { extended }\end{array}$ & Pos/Neg/Neg & 2 & 3.2 & 14.7 & $\begin{array}{l}\text { Cataract; glaucoma; band keratopathy; } \\
\text { synechiae; intraocular calcifications; } \\
\text { chronic hyphema; multiple sub-Tenon } \\
\text { triamcinolone injection. Surgery: } \\
\text { bilateral cataract extraction, left retinal } \\
\text { detachment repair }\end{array}$ & $\begin{array}{r}\text { MTX, LFN, MMF, } \\
\text { CSA, TAC, HCQ, } \\
\text { IVIG, ETN, ADA, } \\
\text { IFX, ABA, ANK }\end{array}$ \\
\hline
\end{tabular}

ABA: abatacept; ADA: adalimumab; ANA: antinuclear antigen; ANK: anakinra; AZA: azathioprine; CSA: cyclosporine; CZP: certolizumab pegol; DMARD: disease-modifying antirheumatic drugs; ETN: etanercept; HCQ: hydroxychloroquine; ILAR: International League of Associations for Rheumatology; IFX: infliximab; IVIG: intravenous immunoglobulin; LFN: leflunomide; MMF: mycophenolate mofetil; MTX: methotrexate; RF: rheumatoid factor; RTX: rituximab; TAC: tacrolimus; Undiffer.: undifferentiated. 
Table 2. Outcome of JIA patients with associated uveitis after switching from TCZ-IV to TCZ-SC treatment.

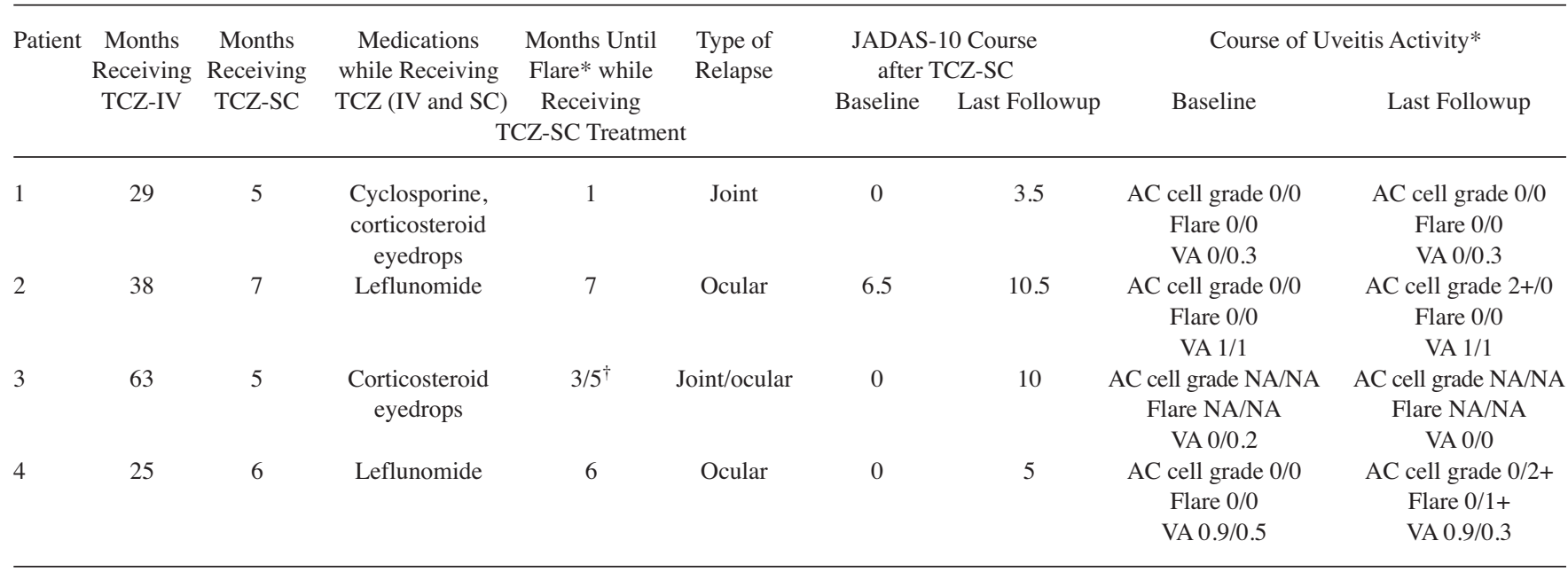

* Uveitis activity determined according to Standardization Uveitis Working Group (SUN) criteria ${ }^{10}$. Ocular flare was defined according to the definition provided by SUN as "two-step increase in level of inflammation or increase from grade 3+ to 4+." Joint flare was based on the already published preliminary definition of flare as "worsening in any $2 / 6$ core response variables (CRV) by $\geq 40 \%$ without concomitant improvement of more than one of the remaining $\mathrm{CRV}$ by $\geq 30 \% " 11$. The $6 \mathrm{CRV}$ are no. joints with active arthritis, no. joints with limited range of motion, physician global assessment of disease severity, parent or patient global assessment, 1 laboratory marker of inflammation, and the score of a functional assessment tool. ${ }^{\dagger} 3$ months until joint flare, 5 months until ocular flare (vitreous hemorrhage). JIA: juvenile idiopathic arthritis; AC: anterior chamber; JADAS: Juvenile Arthritis Disease Activity Score; NA: nonassessable; TCZ-IV: intravenous tocilizumab; TCZ-SC: subcutaneous TCZ; VA: visual acuity.

understood. Therefore, we could speculate that ocular disease is more readily controlled with TCZ-IV than with TCZ-SC because of high blood levels achieved during the first few days after IV infusion, which would help control ocular inflammation. Further studies with patients with JIA are needed to determine the efficacy of TCZ-SC.

ESTEFANIA QUESADA-MASACHS, MD, Department of Rheumatology, Pediatric Rheumatology Unit, Hospital Universitario Vall d'Hebron; CONSUELO MODESTO CABALLERO, MD, PhD, Department of Rheumatology, Pediatric Rheumatology Unit, Hospital Universitario Vall d'Hebron, Barcelona, Spain. Address correspondence to Dr. C. Modesto Caballero, Hospital Universitario Vall d'Hebron, Unidad de Reumatología (Módulo C), Paseo de la Vall d'Hebron 119-129, 08035, Barcelona, Spain. E-mail: cmodesto@vhebron.net

\section{REFERENCES}

1. Brunner HI, Ruperto N, Zuber Z, Keane C, Harari O, Kenwright A, et al. Efficacy and safety of tocilizumab in patients with polyarticular-course juvenile idiopathic arthritis: results from a phase 3, randomised, double-blind withdrawal trial. Ann Rheum Dis 2015;74:1110-7.

2. Navarro-Millan I, Singh JA, Curtis JR. Systematic review of tocilizumab for rheumatoid arthritis: a new biologic agent targeting the interleukin-6 receptor. Clin Ther 2012;34:788-802.e3.

3. De Benedetti F, Brunner HI, Ruperto N, Kenwright A, Wright S, Calvo I, et al. Randomized trial of tocilizumab in systemic juvenile idiopathic arthritis. N Engl J Med 2012;367:2385-95.

4. Tappeiner C, Heinz C, Ganser G, Heiligenhaus A. Is tocilizumab an effective option for treatment of refractory uveitis associated with juvenile idiopathic arthritis? J Rheumatol 2012;39:1294-5.

5. Adan A, Mesquida M, Llorenc V, Modesto C. Tocilizumab for retinal vasoproliferative tumor secondary to juvenile idiopathic arthritis-associated uveitis: a case report. Graefes Arch Clin Exp Ophthalmol 2014;252:163-4.

6. Nguyen QD, Sadiq MA, Chu DS, Dacey MS, Gallemore RP, Dayani $\mathrm{PN}$, et al. Interim outcomes of the study of safety, tolerability, and bioactivity of tocilizumab in patients with non-infectious uveitis (STOP-UVEITIS). Abstract PA060. [Internet. Accessed November 28, 2016.] Available from: https://secure.aao.org/apps/MeetingArchive/tabid/433/Default.aspx

7. Burmester GR, Rubbert-Roth A, Cantagrel A, Hall S, Leszczynski P, Feldman D, et al. A randomised, double-blind, parallel-group study of the safety and efficacy of subcutaneous tocilizumab versus intravenous tocilizumab in combination with traditional disease-modifying antirheumatic drugs in patients with moderate to severe rheumatoid arthritis (SUMMACTA study). Ann Rheum Dis 2014;73:69-74.

8. Ogata A, Atsumi T, Fukuda T, Hirabayashi Y, Inaba M, Ishiguro N, et al. Sustainable efficacy of switching from intravenous to subcutaneous tocilizumab monotherapy in patients with rheumatoid arthritis. Arthritis Care Res 2015;67:1354-62.

9. Iwamoto N, Fukui S, Umeda M, Nishino A, Nakashima Y, Suzuki T, et al. Evaluation of switching from intravenous to subcutaneous formulation of tocilizumab in patients with rheumatoid arthritis Mod Rheumatol 2016;26:662-6.

10. Jabs DA, Nussenblatt RB, Rosenbaum JT; Standardization of Uveitis Nomenclature (SUN) Working Group. Standardization of uveitis nomenclature for reporting clinical data. Results of the First International Workshop. Am J Ophthalmol 2005;140:509-16.

11. Brunner HI, Lovell DJ, Finck BK, Giannini EH. Preliminary definition of disease flare in juvenile rheumatoid arthritis. J Rheumatol 2002;29:1058-64.

J Rheumatol 2017;44:2; doi:10.3899/jrheum.160908 